\title{
Cerebrovascular Disease in Patients with COVID-19: A Review of the Literature and Case Series
}

\author{
Sujan T. Reddy a, b Tanu Garg ${ }^{d} \quad$ Chintan Shah $^{\mathrm{e}}$ Fábio A. Nascimento ${ }^{\mathrm{e}}$ \\ Rajeel Imran ${ }^{d}$ Peter Kan $^{f} \quad$ Ritvij Bowry ${ }^{\text {, c }}$ Nicole Gonzales ${ }^{a, b}$ \\ Andrew Barreto ${ }^{a, b} \quad$ Abhay Kumar $^{c}$ John Volpi ${ }^{d}$ Vivek Misra $^{d}$ \\ David Chiu ${ }^{d}$ Rajan Gadhia ${ }^{d}$ Sean I. Savitz ${ }^{a, b}$ \\ aDepartment of Neurology, University of Texas Health Science Center at Houston, \\ Houston, TX, USA; ' Institute for Stroke and Cerebrovascular Disease, University of Texas \\ Health Science Center at Houston, Houston, TX, USA; 'Department of Neurosurgery, \\ University of Texas Health Science Center at Houston, Houston, TX, USA; ${ }^{d}$ Department of \\ Neurology, Houston Methodist Neurological Institute, Houston, TX, USA; e Department of \\ Neurology, Baylor College of Medicine, Houston, TX, USA; fDepartment of Neurosurgery, \\ Baylor College of Medicine, Houston, TX, USA
}

\section{Keywords}

Stroke $\cdot$ Cerebrovascular disease $\cdot$ Coronavirus · COVID-19 · SARS-CoV-2 · APLS

\section{Abstract}

COVID-19 has been associated with a hypercoagulable state causing cardiovascular and neurovascular complications. To further characterize cerebrovascular disease (CVD) in COVID-19, we review the current literature of published cases and additionally report the clinical presentation, laboratory and diagnostic testing results of 12 cases with COVID-19 infection and concurrent CVD from two academic medical centers in Houston, TX, USA, between March 1 and May 10, 2020. To date, there are 12 case studies reporting 47 cases of CVD in COVID-19. However, only 4 small case series have described the clinical and laboratory findings in patients 


\section{Case Reports in Neurology}

with COVID-19 and concurrent stroke. Viral neurotropism, endothelial dysfunction, coagulopathy and inflammation are plausible proposed mechanisms of CVD in COVID-19 patients. In our case series of 12 patients, 10 patients had an ischemic stroke, of which 1 suffered hemorrhagic transformation and two had intracerebral hemorrhage. Etiology was determined to be embolic without a clear cause identified in 6 ischemic stroke patients, while the remaining had an identifiable source of stroke. The majority of the patients had elevated inflammatory markers such as D-dimer and interleukin-6. In patients with embolic stroke of unclear etiology, COVID-19 may have played a direct or indirect role in the processes that eventually led to the strokes while in the remaining cases, it is unclear if infection contributed partially or was an incidental finding.

(C) 2020 The Author(s)

Published by S. Karger AG, Basel

\section{Introduction}

The ongoing pandemic caused by coronavirus disease (COVID-19) has been associated with high morbidity and mortality. Moreover, COVID-19 has been associated with a hypercoagulable state causing cardiovascular and neurological complications [1, 2]. To date, few studies have reported cerebrovascular complications in COVID-19 [3, 4] and 4 small case series have described the clinical and laboratory findings in patients with COVID-19 and concurrent stroke [5-8]. We review the current literature of published cases and describe our experience of 12 cases with COVID-19 infection and concurrent cerebrovascular disease (CVD) to highlight the clinical presentation and proposed mechanisms of central nervous system (CNS) involvement by SARS-CoV-2.

\section{Methods}

We reviewed the current literature on the pathophysiology and clinical presentation of the published cases of CVD in COVID-19 patients. Additionally, we performed a retrospective chart review of all hospitalized cases with confirmed COVID-19 infection (SARS-CoV-2 RT-PCR positive) and CVD (ischemic and hemorrhagic stroke) between March 1 and May 10, 2020 seen at two comprehensive stroke centers in Houston, TX, USA. Diagnosis of CVD was confirmed on neuroimaging with CT of the brain. Baseline demographic, clinical, laboratory, diagnostic and imaging findings were extracted by a board-certified or board-eligible neurologist. Stroke etiology, according to the TOAST criteria for ischemic stroke, was determined based on available workup [9]. Four of the 12 cases are described in detail for illustrative purposes and the others are summarized in a tabular format. The study was approved by the institutional review board.

\section{Clinical Presentation in the Published Literature}

According to 1 Chinese cohort of 214 confirmed COVID-19 patients, $36.4 \%$ of the patients developed neurological symptoms. CVD was seen in 6 patients $(n=6 / 214 ; 2.8 \%)$ [4]. Since then, other studies have reported CVD in patients with COVID-19; all reported cases, to our best knowledge, are summarized in Table 1. In an observational study documenting 


\section{Case Reports in Neurology}

neurological manifestations of COVID-19, new-onset CVD was present in 5.9\% of the confirmed COVID-19 patients [10]. Stroke (85\%) was the most common form of CVD in addition to isolated cases of cerebral venous sinus thrombosis (CVST) and intracerebral hemorrhage (ICH). The most common stroke etiology in that cohort was large vessel disease (46\%). Development of CVD was seen early in the illness with an average time of 12 days from time of COVID-19 infection to onset of CVD. New-onset CVD was more common in COVID-19 patients who had underlying cerebrovascular risk factors such as older age ( $>65$ years), hypertension, smoking history, and elevated blood glucose levels. In addition, patients with severe pneumonia (as defined by American Thoracic Society guidelines for community-acquired pneumonia) from COVID-19 infection were also more likely to develop new-onset CVD. [10] Furthermore, a recent pooled analysis demonstrated a prior history of CVD to be associated with 2.5 -fold increased odds of severe COVID-19 infection (i.e., requiring mechanical ventilation, intensive care admission, or death) [17].

One study from the Netherlands reviewing thrombotic complications in ICU patients with COVID-19 identified arterial clots in 3.7\% of the patients, all of which caused strokes confirmed with CT imaging [11]. A small case series from the United Kingdom reported 6 COVID19 patients with strokes secondary to large vessel occlusion in all patients [8]. In 1 case report, a cryptogenic stroke manifested as large vessel occlusion a week after the patient developed respiratory symptoms secondary to COVID-19 [12]. Strokes occurring in elderly patients with risk factors were associated with higher mortality (75\%), as reported in a small case series from New York [5]. More recently, 5 cases of large strokes (mean NIHSS 17) in young patients less than 50 years of age secondary to large vessel occlusion were reported in New York City, all of whom tested positive for COVID-19 [6]. A case report from Spain described an initial presentation of COVID-19 as fatal large MCA stroke in a 36-year-old patient [13]. An observational series from France incidentally identified acute strokes on MRI in 3 COVID-19 patients being evaluated for encephalopathy without focal neurological deficits [14]. Additionally, 3 COVID-19 patients in China progressed to develop bilateral cerebral infarcts in multiple vascular territories later in the course of their illness without focal deficits [7]. Although reports of ICH associated with COVID-19 are scarce, 1 case report demonstrated large ICH with intraventricular hemorrhage and subarachnoid hemorrhage (SAH) in a COVID-19 patient without obvious etiology (i.e., trauma, hypertension, or abnormal coagulation) [15]. Similarly, a case of fatal ICH associated with COVID-19 was reported in Wuhan, China [10]. There was also a case report of a young COVID-19 patient who developed aneurysmal SAH 1 week after developing respiratory symptoms [16].

\section{Proposed Pathophysiology}

Viral Neurotropism

Many coronaviruses have similar structures, infection mechanisms, and neuro-invasive potential [18]. SARS-CoV-1 neuro-invasiveness [19] has been established and may be extrapolated to SARS-CoV-2 given the marked similarity between the two coronaviruses [18]. The detection of SARS-CoV-2 in the cerebrospinal fluid (CSF) of a COVID-19 patient with viral encephalitis [20] and the viral presence in neural and capillary endothelial cells in the brain of a COVID-19 patient on autopsy [21] support the viral neurotropism. The routes for neurotropism may include retrograde axonal transport via nasal cavity through the cribriform plate 


\section{Case Reports in Neurology}

and/or hematogenous spread [21]. In the retrograde route, viruses undergo retrograde axonal transport to reach neuronal cells whereas in the hematogenous route viruses access the CNS by infecting the blood-brain barrier endothelial cells, blood-CSF barrier epithelial cells, or using inflammatory cells as decoy [21]. These viruses can then bind to the human angiotensin-converting enzyme II (ACE II) receptors, which is a receptor for the virus spike protein $[22]$.

Endothelial Dysfunction

A recent report of ICH in a COVID-19 patient highlights another potential etiology - endothelial dysfunction. ACE II receptors are expressed in cerebrovascular endothelial cells and regulate the sympathoadrenal system, vascular autoregulation, and cerebral blood flow [23]. Virus binding to the CNS ACE II receptors can lead to disruption of its autoregulatory function causing blood pressure elevations leading to vessel wall rupture [15].

\section{Coagulopathy}

A prospective study reported that D-dimer, fibrin/fibrinogen degradation products, and fibrinogen values were elevated in patients with COVID-19, whereas anti-thrombin and PT activity were lower compared to healthy controls. D-dimer and fibrin/fibrinogen degradation product values in patients with severe cases were higher compared to patients with milder forms of the infection [24]. The alteration of the coagulation pathway with elevated D-dimer and fibrinogen may also contribute to the increased risk of thrombosis.

Case series have reported the presence of positive anti- $\beta 2$-glycoprotein I IgA and IgG antibodies, but negative lupus anticoagulant in critically ill elderly patients with COVID-19 [7]. These antiphospholipid antibodies have been detected in various viral infections and are associated with thrombosis but are usually transient and not suggestive of antiphospholipid syndrome [25].

Viral infection leading to sepsis with subsequent appearance of disseminated intravascular coagulation (DIC) has also been indirectly implicated as a potential cause of COVID-19associated thrombosis. Monocytes and endothelial cell activation leading to cytokine release can result in DIC development along with tissue factor expression and von Willebrand factor secretion [26]. Free thrombin circulation without full control by natural anticoagulants can both activate platelets and cause fibrinolysis [27]. The link between DIC and stroke is still under speculation but is a considerable hypothesis.

Inflammation

Cytokines have also been implicated in the pathogenicity of this virus - through a systemic inflammatory response syndrome and induction of a pro-inflammatory state [28] leading to a cytokine storm. In the REGARDS cohort study, higher levels of circulating IL- 6 were associated with a significantly increased risk of incident stroke independent of stroke risk factors [29]. Given the importance of increased levels of IL-6 in both COVID-19 symptom severity and increased risk of incident stroke in the general population, it is possible that IL- 6 may be playing a key role in COVID-19 patients and concomitant stroke. Pro-inflammatory interleukin receptor antibodies are being considered as potential treatment options for certain subset of patients [30]. 


\section{Case Reports in Neurology}

Another potential mechanism includes cardio-embolism from virus-associated myocardial injury. Viral spread associated cytokine storm and subsequent immune responses may contribute to myocardial injury by decreasing coronary blood flow, oxygen supply, destabilization of coronary plaque, and micro-thrombogenesis [31]. Lastly, virus proliferation in the lung can cause hypoxia, which can lead to stroke - especially in patients already at risk of developing CVD [28].

\section{Case Series}

We describe a total of 12 patients with COVID-19 and concurrent CVD. Ten patients were diagnosed with acute ischemic stroke and two with ICH. Individual patient level data including clinical, laboratory and diagnostic results are summarized in online supplementary Table 1 (for all online suppl. material, see www.karger.com.doi/10.1159/000508958). Six patients (patients 1, 2, 3, 8, 9, and 12) were diagnosed with stroke of undetermined etiology based on TOAST criteria. The etiology for patient 5 was determined to be cardioembolic, that for patient 7 was carotid dissection, and that for patient 10 was infectious endocarditis. Workup for patient 6 was incomplete due to isolation precautions. One hemorrhagic stroke (patient 4) was likely hypertensive and another was related to mycotic aneurysm from infectious endocarditis (patient 11).

\section{Patient 1}

A 59-year-old woman was transferred from another facility for CT brain findings of subacute left frontal and occipital infarcts found on day 14 after initial admission for COVID-19related acute respiratory distress syndrome (ARDS) (Fig. 1a). CTA of the brain was normal. She was also found to have extensive bilateral deep venous thrombi (DVT). Transthoracic ultrasonography was negative for patent foramen ovale. She was treated with hydroxychloroquine and azithromycin for COVID-ARDS and anticoagulation for DVT and eventually discharged to the inpatient rehabilitation unit.

\section{Patient 2}

A 47-year-old man presented with $24 \mathrm{~h}$ of left hemiplegia. CT of the brain showed right parietal, temporal and occipital infarctions (Fig. 1b) and vascular imaging showed occlusion of the right middle cerebral artery but no carotid disease. The admission chest X-ray showed bibasilar opacities and COVID-19 testing was positive. Neurological symptom onset was on day 7 of respiratory symptoms with fever and cough. Transthoracic echocardiogram was unrevealing. He was treated with hydroxychloroquine and azithromycin and discharged home on antiplatelet therapy for stroke of undetermined etiology after two negative repeat COVID19 tests and unrevealing stroke workup.

\section{Patient 3}

A 39-year-old-woman was hospitalized for COVID-19 pneumonia treatment. On day 7 of hospitalization, she developed right hemiparesis. CT of the brain showed early ischemic changes in the left frontal lobe (Fig. 1c). CTA of the brain showed left middle cerebral artery occlusion, but no proximal arterial disease and she was successfully re-perfused with 


\section{Case Reports in Neurology}

intravenous thrombolysis and mechanical thrombectomy. Workup revealed elevated anticardiolipin antibodies but she did not meet the criteria for anti-phospholipid syndrome [32]. She was discharged home on antiplatelet therapy for stroke of undetermined etiology.

\section{Patient 4}

A 48-year-old man presented with left hemiparesis and blood pressure of 259/151. CT of the brain showed dorsolateral pontine hemorrhage atypical in location for a hypertensive hemorrhage (Fig. 1d). CTA of the brain was normal. Chest X-ray showed bilateral infra-hilar opacities representing infection or aspiration and labs consistent with COVID infection. 3,4 A positive serum rapid plasma reagin test with negative treponemal antibody testing, unrevealing CSF analysis and negative CSF VDRL testing prompted the evaluation for falsely positive rapid plasma reagin test that revealed elevated anti-cardiolipin antibodies and $\beta 2$ glycoprotein antibodies (online suppl. Table 1). On day 20, COVID-19 testing was positive. He remained asymptomatic throughout without fever or cough and was discharged home with instructions for self-quarantine, outpatient gadolinium-enhanced MRI of the brain, tele-stroke clinic and hematology follow-up.

\section{Discussion}

Six of our 10 ischemic stroke patients were found to have strokes of undetermined etiology, which could potentially be attributable to a hypercoagulable state from COVID-19 infection. All our patients had elevated D-dimer on admission in the setting of COVID-19 like syndrome with elevated LDH levels and lymphopenia [33, 34]. While elevated D-dimer levels are suggestive of hypercoagulability, they do not establish causality between CVD and COVID-19. A consensus statement by Zhai et al. [35] suggests while D-dimer levels in the early course of COVID-19 pneumonia could indicate inflammatory response, it can also represent acute thrombosis and monitoring of D-dimer, fibrinogen and fibrin degradation product values may be useful for the timely identification of a thrombotic complication. COVID-19 patients with D-dimer levels $\geq 2.0 \mu \mathrm{g} / \mathrm{ml}$ have a higher incidence of mortality [1]. Notably, a study by Ageno et al. [36] showed that D-dimer levels were significantly higher in cardioembolic etiologies compared to lacunar etiologies of strokes. Three of the 6 patients with strokes of undetermined etiology in our series were relatively young. Younger black and Hispanics have been shown to have a greater incidence of stroke [37] and strikingly, our young patients consisted of black, Hispanic and Asian ethnicities.

The other population in our series represented patients with a traditional, identifiable cause of stroke. It remains unclear if COVID-19 associated hypercoagulability increased the risk of thrombosis in these patients.

One patient in our series developed an ICH with positive antiphospholipid antibodies. Antiphospholipid syndrome in COVID-19 has previously been described [7]. However, it is uncertain if it represents an acute-phase reaction or a hypercoagulable state [38]. While anticardiolipin antibodies were also elevated in one other patient in our series, neither met criteria for anti-phospholipid syndrome [32]. In our patient with ICH, COVID-19 could be unrelated in this case and hypertension could be the etiology, although hypertensive hemorrhages typically occur centrally within the pons. 


\section{Case Reports in Neurology}

Our literature review and case series raise the importance for registries on the incidence and timing of cerebrovascular complications in patients with COVID-19 to better understand the natural history and help inform and improve patient management of COVID-associated CVD. As an example, there is controversy on empiric systemic anticoagulation in patients with COVID-19 presenting with elevated d-dimers given the high incidence of thromboembolic complications [38, 39] and higher odds of mortality with high d-dimer values [40]. Which patients would benefit from preventative and therapeutic anticoagulation or antiplatelet agents in COVID patients at risk for CVD awaits further data. In conclusion, the relationship between CVD and COVID-19 seems twofold: CVD seems to be relatively common in patients with COVID-19 who develop neurological complications [4] and having a history of CVD and its risk factors appears to be more common in patients with a severe form of COVID-19 [17]. Detection of CVD in severe COVID-19 infections has been challenging given the lack of focal neurological deficits and limited examination in many critical care patients. The pathophysiology of CVD in patients with concomitant COVID-19 infection is still a topic of debate. Potential contenders include direct invasion of the brain, endothelial dysfunction, hypercoagulable state, cardio-embolism, cytokine storm, and hypoxia. Understanding the relationship between CVD and COVID-19 is of paramount importance to implement management changes and provide adequate care for this patient population.

\section{Statement of Ethics}

The research was conducted ethically in accordance with the World Medical Association Declaration of Helsinki. Written informed consent was not obtained due to the retrospective nature of case series and was waived by approval from the institutional review board committee on human research.

\section{Disclosure Statement}

F. Nascimento is a member of the Neurology Resident and Fellow Section Editorial Team. The other authors have no conflicts of interest to declare.

\section{Funding Sources}

NIH Stroke Net grant funding (U24 NS107322) was given to Sujan T. Reddy.

\section{Author Contributions}

Sujan T. Reddy: data acquisition and analysis, drafting, revision of manuscript.

Fábio A. Nascimento: data analysis and interpretation, drafting of the manuscript.

Chintan Shah: data analysis and interpretation, drafting of the manuscript. 
Reddy et al.: Stroke in COVID-19

Peter Kan: data analysis and interpretation, revising manuscript, study supervision, final approval.

Rajeel Imran: data acquisition, drafting, revision of the manuscript.

Tanu Garg: data acquisition, drafting, revision of the manuscript.

Ritvij Bowry: data acquisition, drafting, revision of the manuscript.

Nicole Gonzales: data acquisition, drafting, revision of the manuscript.

Andrew Barreto: data acquisition, drafting, revision of the manuscript.

Abhay Kumar: data acquisition, drafting, revision of the manuscript.

John Volpi: drafting, revision of the manuscript.

Vivek Misra: data acquisition, drafting, revision of the manuscript.

David Chiu: data acquisition, drafting, revision of the manuscript.

Rajan Gadhia: data acquisition, drafting, revision of the manuscript.

Sean I. Savitz: drafting, revision of the manuscript.

All authors accept responsibility for the conduct of the research.

\section{References}

1 Zhang L, Yan X, Fan Q, Liu H, Liu X, Liu Z, et al. D-dimer levels on admission to predict in-hospital mortality in patients with Covid-19. J Thromb Haemost. 2020 Apr;jth.14859.

2 Bangalore S, Sharma A, Slotwiner A, Yatskar L, Harari R, Shah B, et al. ST-Segment Elevation in Patients with Covid-19 - A Case Series. N Engl J Med. 2020 Apr;NEJMc2009020.

3 Huang C, Wang Y, Li X, Ren L, Zhao J, Hu Y, et al. Clinical features of patients infected with 2019 novel coronavirus in Wuhan, China. Lancet. 2020 Feb;395(10223):497-506.

4 Mao L, Jin H, Wang M, Hu Y, Chen S, He Q, et al. Neurologic Manifestations of Hospitalized Patients With Coronavirus Disease 2019 in Wuhan, China. JAMA Neurol. 2020 Apr 10;e201127.

5 Avula A, Nalleballe K, Narula N, Sapozhnikov S, Dandu V, Toom S, et al. COVID-19 presenting as stroke. Brain Behav Immun. 2020 Apr;S0889-1591(20)30685-1.

6 Oxley TJ, Mocco J, Majidi S, Kellner CP, Shoirah H, Singh IP, et al. Large-Vessel Stroke as a Presenting Feature of Covid-19 in the Young. N Engl J Med. 2020 May;382(20):e60.

7 Zhang Y, Xiao M, Zhang S, Xia P, Cao W, Jiang W, et al. Coagulopathy and Antiphospholipid Antibodies in Patients with Covid-19. N Engl J Med. 2020 Apr;382(17):e38.

8 Beyrouti R, Adams ME, Benjamin L, Cohen H, Farmer SF, Goh YY, et al. Characteristics of ischaemic stroke associated with COVID-19. J Neurol Neurosurg Psychiatry. 2020 Apr;jnnp-2020-323586.

9 Adams HP Jr, Bendixen BH, Kappelle LJ, Biller J, Love BB, Gordon DL, et al. Classification of subtype of acute ischemic stroke. Definitions for use in a multicenter clinical trial. TOAST. Trial of Org 10172 in Acute Stroke Treatment. Stroke. 1993 Jan;24(1):35-41.

10 Li Y, Wang M, Zhou Y, Chan Y, Xian Y, Mao L, et al. Acute cerebrovascular disease following COVID-19: a single center, retrospective, observational study [cited 2020 Mar 3]. Available from: https://ssrncom/abstract=3550025.

11 Klok FA, Kruip M, van der Meer NJ, Arbous MS, Gommers D, Kant KM, et al. Incidence of thrombotic complications in critically ill ICU patients with COVID-19. Thromb Res. 2020 Jul;191:145-7.

12 Valderrama EV, Humbert K, Lord A, Frontera J, Yaghi S. Severe Acute Respiratory Syndrome Coronavirus 2 Infection and Ischemic Stroke. Stroke. 2020:Strokeaha120030153.

13 González-Pinto T, Luna-Rodríguez A, Moreno-Estébanez A, Agirre-Beitia G, Rodríguez-Antigüedad A, RuizLopez M. Emergency room neurology in times of COVID-19: malignant ischaemic stroke and SARS-CoV-2 infection. Eur J Neurol. 2020 Apr;ene.14286.

14 Helms J, Kremer S, Merdji H, Clere-Jehl R, Schenck M, Kummerlen C, et al. Neurologic Features in Severe SARS-CoV-2 Infection. N Engl J Med. 2020 Jun 4;382(23):2268-70.

15 Sharifi-Razavi A, Karimi N, Rouhani N. COVID-19 and intracerebral haemorrhage: causative or coincidental? New Microbes New Infect. 2020 Mar 27;35:100669. 
Reddy et al.: Stroke in COVID-19

16 Al Saiegh F, Ghosh R, Leibold A, Avery MB, Schmidt RF, Theofanis T, et al. Status of SARS-CoV-2 in cerebrospinal fluid of patients with COVID-19 and stroke. J Neurol Neurosurg Psychiatry. 2020 Apr;jnnp2020-323522.

17 Aggarwal G, Lippi G, Michael Henry B. Cerebrovascular disease is associated with an increased disease severity in patients with Coronavirus Disease 2019 (COVID-19): A pooled analysis of published literature. Int J Stroke. 2020 Jun;15(4):385-9.

18 Li YC, Bai WZ, Hashikawa T. The neuroinvasive potential of SARS-CoV2 may play a role in the respiratory failure of COVID-19 patients. J Med Virol. 2020 Feb.

19 Glass WG, Subbarao K, Murphy B, Murphy PM. Mechanisms of host defense following severe acute respiratory syndrome-coronavirus (SARS-CoV) pulmonary infection of mice. J Immunol. 2004 Sep;173(6):4030-9.

20 Moriguchi T, Harii N, Goto J, Harada D, Sugawara H, Takamino J, et al. A first case of meningitis/encephalitis associated with SARS-Coronavirus-2. Int J Infect Dis. 2020 May;94:55-8.

21 Paniz-Mondolfi A, Bryce C, Grimes Z, Gordon RE, Reidy J, Lednicky J, et al. Central Nervous System Involvement by Severe Acute Respiratory Syndrome Coronavirus -2 (SARS-CoV-2). J Med Virol. 2020 Apr;jmv.25915.

22 Baig AM, Khaleeq A, Ali U, Syeda H. Evidence of the COVID-19 Virus Targeting the CNS: Tissue Distribution, Host-Virus Interaction, and Proposed Neurotropic Mechanisms. ACS Chem Neurosci. 2020 Apr;11(7):995-8.

23 Saavedra JM. Brain angiotensin II: new developments, unanswered questions and therapeutic opportunities. Cell Mol Neurobiol. 2005 Jun;25(3-4):485-512.

24 Han H, Yang L, Liu R, Liu F, Wu KL, Li J, et al. Prominent changes in blood coagulation of patients with SARSCoV-2 infection. Clin Chem Lab Med. 2020 Mar;0(0):/j/cclm.ahead-of-print/cclm-2020-0188/cclm-20200188.xml.

25 Uthman IW, Gharavi AE. Viral infections and antiphospholipid antibodies. Semin Arthritis Rheum. 2002 Feb;31(4):256-63.

26 Tang N, Li D, Wang X, Sun Z. Abnormal coagulation parameters are associated with poor prognosis in patients with novel coronavirus pneumonia. J Thromb Haemost. 2020 Apr;18(4):844-7.

27 Kitchens CS. Thrombocytopenia and thrombosis in disseminated intravascular coagulation (DIC). Hematology (Am Soc Hematol Educ Program). 2009;2009(1):240-6.

$28 \mathrm{Wu}$ Y, Xu X, Chen Z, Duan J, Hashimoto K, Yang L, et al. Nervous system involvement after infection with COVID-19 and other coronaviruses. Brain Behav Immun. 2020 Mar;S0889-1591(20)30357-3.

29 Jenny NS, Callas PW, Judd SE, McClure LA, Kissela B, Zakai NA, et al. Inflammatory cytokines and ischemic stroke risk: the REGARDS cohort. Neurology. 2019 May;92(20):e2375-84.

30 Luo P, Liu Y, Qiu L, Liu X, Liu D, Li J. Tocilizumab treatment in COVID-19: A single center experience. J Med Virol. 2020 Apr. https://doi.org/10.1002/jmv.25801.

31 Guo T, Fan Y, Chen M, Wu X, Zhang L, He T, et al. Cardiovascular Implications of Fatal Outcomes of Patients With Coronavirus Disease 2019 (COVID-19). JAMA Cardiol. 2020 Mar. https://doi.org/10.1001/jamacardio.2020.1017.

32 Bustamante JG, Goyal A, Bansal P, Singhal M. Antiphospholipid Syndrome (Antiphospholipid Antibody Syndrome, APS, APLS). StatPearls. Treasure Island (FL): StatPearls Publishing StatPearls Publishing LLC; 2020.

33 Tan L, Wang Q, Zhang D, Ding J, Huang Q, Tang YQ, et al. Lymphopenia predicts disease severity of COVID-19: a descriptive and predictive study. Signal Transduct Target Ther. 2020 Mar 27;5(1):33.

34 Terpos E, Ntanasis-Stathopoulos I, Elalamy I, Kastritis E, Sergentanis TN, Politou M, et al. Hematological findings and complications of COVID-19. Am J Hematol. 2020 Apr;ajh.25829.

35 Zhai Z, Li C, Chen Y, Gerotziafas G, Zhang Z, Wan J, et al.; Prevention Treatment of VTE Associated with COVID-19 Infection Consensus Statement Group. Prevention and Treatment of Venous Thromboembolism Associated with Coronavirus Disease 2019 Infection: A Consensus Statement before Guidelines. Thromb Haemost. 2020 Apr. https://doi.org/10.1055/s-0040-1710019.

36 Ageno W, Finazzi S, Steidl L, Biotti MG, Mera V, Melzi D’Eril G, et al. Plasma measurement of D-dimer levels for the early diagnosis of ischemic stroke subtypes. Arch Intern Med. 2002 Dec;162(22):2589-93.

37 Jacobs BS, Boden-Albala B, Lin IF, Sacco RL. Stroke in the young in the northern Manhattan stroke study. Stroke. 2002 Dec;33(12):2789-93.

38 Bikdeli B, Madhavan MV, Jimenez D, Chuich T, Dreyfus I, Driggin E, et al. COVID-19 and Thrombotic or Thromboembolic Disease: Implications for Prevention, Antithrombotic Therapy, and Follow-up. J Am Coll Cardiol. 2020 Apr;S0735-1097(20)35008-7.

39 Tang N, Bai H, Chen X, Gong J, Li D, Sun Z. Anticoagulant treatment is associated with decreased mortality in severe coronavirus disease 2019 patients with coagulopathy. J Thromb Haemost. 2020 May;18(5):1094-9. 


\section{Case Reports in Neurology}

\begin{tabular}{l|l} 
Case Rep Neurol 2020;12:199-209 \\
\hline DOI: 10.1159/000508958 & $\begin{array}{l}\text { (c) 2020 The Author(s). Published by S. Karger AG, Basel } \\
\text { www.karger.com/crn }\end{array}$
\end{tabular}

Reddy et al.: Stroke in COVID-19

40 Rodelo JR, De la Rosa G, Valencia ML, Ospina S, Arango CM, Gómez CI, et al. D-dimer is a significant

prognostic factor in patients with suspected infection and sepsis. Am J Emerg Med. 2012 Nov;30(9):1991-9.
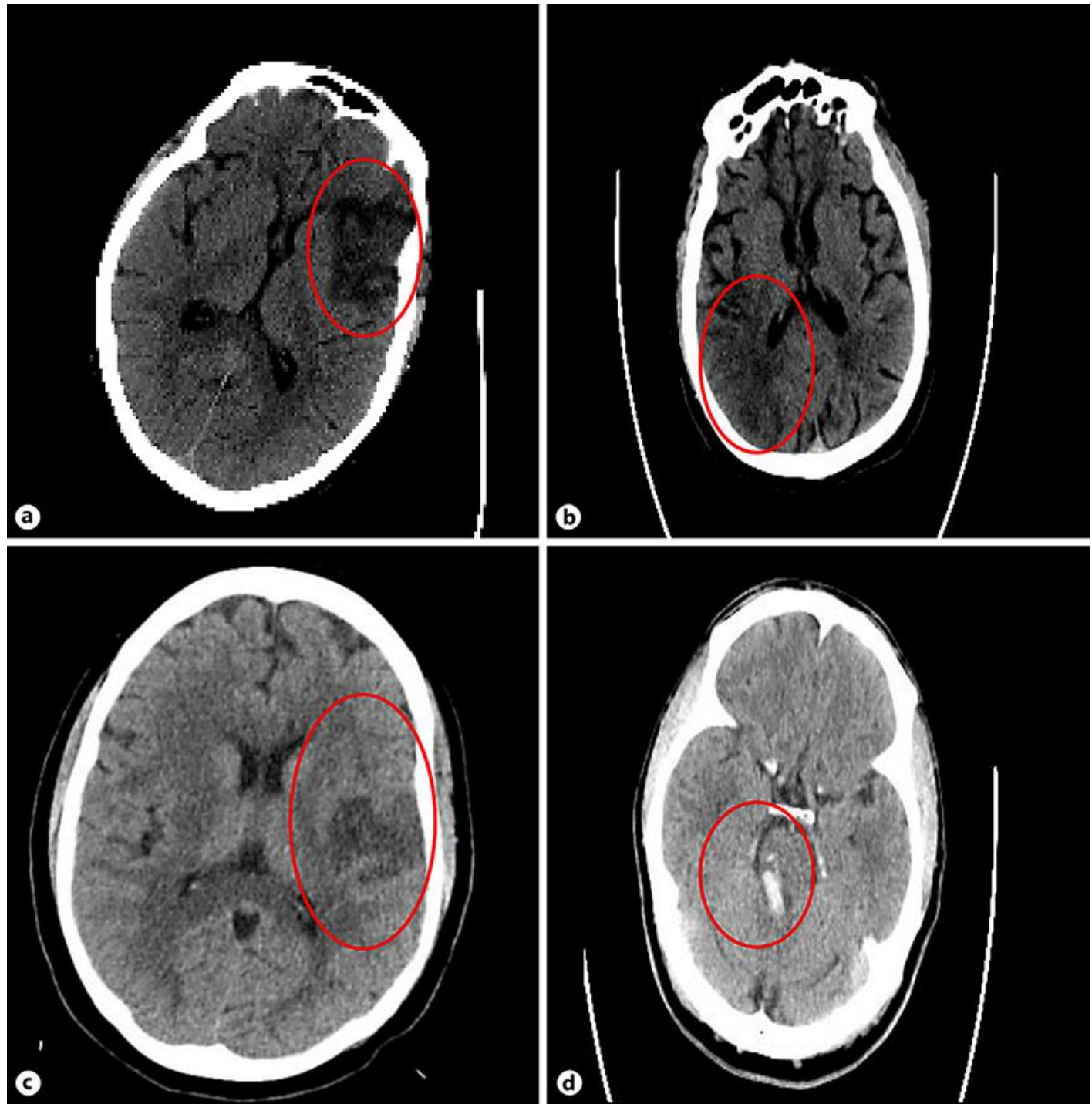

Fig. 1. a CT brain images ofan illustrative case (patient 1). b CT brain images of an illustrative case (patient 2). c CT brain images of an illustrative case (patient 3). d CT brain images of an illustrative case (patient 4). 
Table 1. Summary of published cases of cerebrovascular disease in COVID-19

\begin{tabular}{llllll}
\hline Study & $\begin{array}{l}\text { Total COVID-19, } \\
n\end{array}$ & $\begin{array}{l}\text { Patients with } \\
\text { CVD, } n(\%)\end{array}$ & CVD subtype & Etiology & $\begin{array}{l}\text { Mortality } \\
\text { rate, } \%\end{array}$ \\
\hline Maoa et al. [4] & 214 & $\begin{array}{l}5(2.3) \\
1(0.5)\end{array}$ & $\begin{array}{l}\text { Stroke } \\
\text { ICH }\end{array}$ & $\begin{array}{l}\text { NR } \\
\text { NR }\end{array}$ & $\begin{array}{l}\text { NR } \\
100 \%\end{array}$ \\
\hline Lia et al. [10] & 221 & $11(5)$ & Stroke & $\begin{array}{l}46 \% \text { LVD } \\
27 \% \text { SVD }\end{array}$ & $36 \%$ \\
& & & & $27 \%$ CE & \\
& & $1(0.5)$ & CVST & \\
\hline Klok et al. [11] & 184 & ICH & Stroke & NR & NR \\
\hline Beyrouti et al. [8] & 6 & $3(1.6)$ & Stroke & NR & $17 \%$ \\
\hline Valderrama et al. [12] & 1 Stroke & $1(100)$ & Stroke & Cryptogenic & $0 \%$ \\
\hline Avula et al. [5] & 4 & $4(100)$ & Stroke & NR & $75 \%$ \\
\hline Oxley et al. [6] & 5 & $5(100)$ & Stroke & NR & $0 \%$ \\
\hline González-Pinto et al. [13] & 1 & $1(100)$ & Stroke & NR & $100 \%$ \\
\hline Helms et al. [14] & 58 & $3(5.2)$ & Stroke & NR & NR \\
\hline Zhang et al. [7] & 3 & $3(100)$ & Stroke & NR & NR \\
\hline Sharifi-Razavi et al. [15] & 1 & $1(100)$ & ICH & NR & NR \\
\hline Al Saiegh et al. [16] & 1 & $1(100)$ & SAH & Aneurysmal & $0 \%$ \\
\hline
\end{tabular}

CVD, cerebrovascular disease; CVST, cerebral venous sinus thrombosis; ICH, intracranial hemorrhage; LVD, large vessel disease; SVD, small vessel disease; CE, cardio-embolism; NR, not reported; SAH, subarachnoid hemorrhage. a Overlapping data: both case series represent patients analyzed within the same time window from the same center. 